\title{
Existence, Decay, and Blow-Up of Solutions for a Higher-Order Kirchhoff-Type Equation with Delay Term
}

\author{
Hazal Yüksekkaya, ${ }^{1}$ Erhan Pișkin, ${ }^{1}$ Salah Mahmoud Boulaaras $\mathbb{D}^{2,3}$ \\ and Bahri Belkacem Cherif $\mathbb{i D}^{2,4}$ \\ ${ }^{1}$ Department of Mathematics, Dicle University, Diyarbakir, Turkey \\ ${ }^{2}$ Department of Mathematics, College of Sciences and Arts, Qassim University, Ar Rass, Saudi Arabia \\ ${ }^{3}$ Laboratory of Fundamental and Applied Mathematics of Oran (LMFAO), University of Oran, 1, Oran, 31000 Oran, Algeria \\ ${ }^{4}$ Preparatory Institute for Engineering Studies in Sfax, Tunisia
}

Correspondence should be addressed to Salah Mahmoud Boulaaras; s.boularas@qu.edu.sa

Received 19 April 2021; Accepted 18 August 2021; Published 2 September 2021

Academic Editor: Gangadharan Murugusundaramoorthy

Copyright (c) 2021 Hazal Yüksekkaya et al. This is an open access article distributed under the Creative Commons Attribution License, which permits unrestricted use, distribution, and reproduction in any medium, provided the original work is properly cited.

\begin{abstract}
This article deals with the study of the higher-order Kirchhoff-type equation with delay term in a bounded domain with initial boundary conditions, where firstly, we prove the global existence result of the solution. Then, we discuss the decay of solutions by using Nakao's technique and denote polynomially and exponentially. Furthermore, the blow-up result is established for negative initial energy under appropriate conditions.
\end{abstract}

\section{Introduction}

In this paper, we establish the higher-order Kirchhoff-type equation with delay term as follows:

$$
\begin{cases}u_{t t}+\left(\int_{\Omega}\left|A^{m / 2} u\right|^{2} d x\right)^{q} A^{m} u+\mu_{1}\left|\mu_{t}(x, t)\right|^{r-1} \mu_{t}(x, t) & \\ +\mu_{2}\left|\mu_{t}(x, t-\tau)\right|^{r-1} u_{t}(x, t-\tau)=|u|^{p-1} u, & (x, t) \in \Omega \times(0, T), \\ u(x, 0)=u_{0}(x), u_{t}(x, 0)=u_{1}(x) & x \in \Omega \\ u_{t}(x, t-\tau)=f_{0}(x, t-\tau) & x \in \Omega, t \in(0, \tau), \\ \frac{\partial^{i} u}{\partial v^{i}}=0, i=0,1, \cdots, m-1 & x \in \partial \Omega,\end{cases}
$$

where $A=-\Delta, m \geq 1$ is a natural number, $q, r \geq 0$ are real numbers, $p>1$ is a real number and is a bounded domain with smooth boundary $\partial \Omega$ in $R^{n}, n=1 ; 2 ; 3 ; v$ is the outer normal. $\tau>0$ denotes time delay, and ${ }_{1}$ and ${ }_{2}$ are positive real numbers. The functions $\left(u_{0}, u_{1}, f_{0}\right)$ are the initial data belong to a suitable space.
The problem (1) is a general form of a model introduced by Kirchhoff [1]. To be more precise, Kirchhoff recommended a model denoted by the equation for $f=g=0$,

$p h \frac{\partial^{2} u}{\partial t^{2}}+\delta \frac{\partial u}{\partial t}+g\left(\frac{\partial u}{\partial t}\right)=\left\{\rho_{0}+\frac{E h}{2 L} \int_{0}^{L}\left(\frac{\partial u}{\partial x}\right)^{2} d x\right\} \frac{\partial^{2} u}{\partial x^{2}}+f(u)$,

for $0<x<L, t \geq 0$, where $u(x, t)$ is the lateral displacement, $\rho$ is the mass density, $h$ is the cross-section area, $E$ is the Young modulus, $L$ is the length, $\rho_{0}$ is the initial axial tension, and $f, g$ are the external forces. Furthermore, (2) is called a degenerate equation when $\rho_{0}=0$ and nondegenerate one when $\rho_{0}>0$.

Time delays often appear in many various problems, such as thermal, economic phenomena, biological, chemical, and physical. Recently, the partial di/erential equations with time delay have become an active area, (see $[2,3]$ and references therein). Datko et al. [4] indicated that a small delay in a boundary control is a source of instability. An arbitrarily small delay may destabilize a system which is uniformly 
asymptotically stable without delay unless additional conditions or control terms have been used in many cases [5]. Additional control terms will be necessary to stabilize hyperbolic systems including delay terms, (see [6-8] and references therein). In [6], Nicaise and Pignotti studied the equation as follows:

$$
u_{t t}(x, t)-\Delta u(x, t)+a_{0} u_{t}(x, t)+a u_{t}(x, t-\tau)=0,
$$

where $a_{0}$ and $a$ are positive real parameters. The authors obtained that, under the condition $0 \leq \alpha \leq a_{0}$, the system is exponentially stable. In the case $\alpha \geq \alpha$, they obtained a sequence of delays that shows the solution is instable. In [8], $\mathrm{Xu}$ et al. obtained the same result similar to the [6] for the one space dimension by adopting the spectral analysis approach. In [9], Nicaise et al. studied the wave equation in one space dimension in the case of time-varying delay. In that work, the authors showed that an exponential stability result under the condition

$$
\alpha \leq \sqrt{1-d \alpha_{0}}
$$

where $d$ is a constant such that

$$
\tau^{\prime}(t) \leq d<1, \forall t>0
$$

In recent years, some other authors investigate hyperbolic type equation with delay term (see [10-16]).

Without delay term $\left(\mu_{2}\left|u_{t}(x, t-\tau)\right|^{r-1} u_{t}(x, t-\tau)\right)$, in 2004, Li [17] studied the higher-order Kirchho/-type equation as follows:

$$
\mu_{t t}+\left(\int_{\Omega}\left|D^{m / 2} u\right|^{2} d x\right)^{q}(-\Delta)^{m} u+u_{t}\left|u_{t}\right|^{r}=|u|^{p} u
$$

where $m>1$ is a positive integer, and $q, p, r>0$ is a positive constant. The author obtained that the solution exists globally if $p \leq r$, while if $p>\max \{r, 2 q\}$. He also established the blow-up result for $E(0)<0$. Later, in 2007, Messaoudi and Houari [18] obtained the blow-up of solutions with $E$ ( $0)>0$ of the equation (6). Then, Piskin and Polat [19] considered global existence and decay estimates utilizing Nakao's inequality of the equation (6).

Without delay term, when $m=1$ and $q=0$, equation (1) takes the form of a semilinear hyperbolic equation as follows:

$$
u_{t t}-\Delta u+u_{t}\left|u_{t}\right|^{r-1}=|u|^{p-1} u
$$

Georgiev and Todorova [20] obtained the blow-up of solutions for $E(0)<0$ if $1<r<p(1<p<n /(n-2)$ for $n \geq 3$, $p>1$ for $n<3$ ) of the equation (7). Under the condition of positive upper bounded initial energy, Vitillaro [21] proved the same results of equation (7). Also, Ohta [22, 23] studied related problems for the blow-up results of the equation (7).

Messaoudi [24] studied the following equation

$$
u_{t t}+\Delta^{2} u+\left|u_{t}\right|^{r 2} u_{t}=|u|^{p 2} u
$$

and obtained an existence result for the equation (8) and proved that the solution continues to exists globally if $r \geq p$; however, if $r<p$ and the initial energy is negative, the solution blows up in finite time. Chen [25] established that the solution of (8) blows up with $E(0)>0$. In the presence of strong damping term $\left(-\Delta u_{t}\right)$, Piskin and Polat [26] obtained the decay estimates by using Nakao's inequality of equation (8).

When $m=1$ and without delay term, equation (1) takes the form the following Kirchhoff-type equation:

$$
u_{t t^{-}}\left(\int_{\Omega}\left|D_{u}\right|^{2} d x\right)^{\gamma} \Delta_{u}+u_{t}\left|u_{t}\right|^{r}=|u|^{p} u
$$

Many authors had studied existence and blow-up results at night time for equation (9) (see [27-30]). Ono [30] proved the blow-up results if $p>\max \{r, 2 \gamma\}(p<2 /(n-4)$ for $n \geq 5$ $, p>0$ for $n \leq 4)$ and $E(0)<0$ for equation (9). Later, Benaissa and Messaoudi [31] obtained the similar result for the generalized Kirchhoff-type equation as follows:

$u_{t t}-M\left(\int_{\Omega} e^{\phi(x)}|\nabla u|^{2} d x\right) e^{-\phi(x)} \operatorname{div}\left(e^{\phi(x)} \nabla u\right)+\alpha\left|u_{t}\right|^{r-2} u_{t}=b|u|^{p-2} u$

where $M: R^{+} \longrightarrow R^{+}$and $\phi(x)$ are bounded functions. Then, $\mathrm{Wu}$ [32], verified the same result of the general Kirchhoff-type equation

$$
u_{t t}-M\left(\|\nabla u\|_{2}^{2}\right) \Delta u+\left|u_{t}\right|^{r-2} u_{t}=|u|^{p-2} u,
$$

with the positive upper bounded initial energy. In 2013, Ye [33] considered the global existence results by constructing a stable set in $H_{0}^{1}(\Omega)$ and showed the decay by using a lemma of Komornik for the nonlinear Kirchhoff-type equation (11) with dissipative term. Moreover, Ye [34] obtained the global existence results by constructing a stable set in $H_{0}^{m}(\Omega)$ and showed the energy decay by using a lemma of $V$. Komornik for a nonlinear higher-order Kirchhoff-type equation with dissipative term is as follows:

$$
u_{t t}+\left\|A^{1 / 2} u\right\|^{2 p} A u+a\left|u_{t}\right|^{r-2} u_{t}=b|u|^{p-2} u
$$

where $A=(-\Delta)^{m}, m>1$ is a positive integer.

Gao et al. [35] considered the Kirchhoff-type equation without delay term as follows:

$$
u_{t t}+M\left(\left\|D^{m} u\right\|_{2}^{2}\right)(-\Delta)^{m} u+\left|u_{t}\right|^{r-2} u_{t}=|u|^{p-2} u
$$

The authors obtained the blow-up of solutions for $E(0)$ $>0$ under appropriate conditions for equation (13).

In [36-40], some authors studied abstract evolution equations as follows:

$$
\left[P\left(u_{t}\right)\right]_{t} A(t, u)+Q\left(t, u_{t}\right)=F(u)
$$

on suitable Banach space, and they proved some global 
nonexistence of solutions. Some other authors studied related problems (see [41-45]).

Motivated by the above works, we deal with the existence, decay, and blow-up results for the higher-order Kirchhoff type equation (1) with delay term and source term. There is no research, to our best knowledge, related to the higher-order Kirchhoff-type $\left(\left(\int_{\Omega}\left|A^{m / 2} u\right|^{2} d x\right)^{q} A^{m} u\right)$ equation (1) with delay $\left(u_{2}\left|u_{t}(x, t-\tau)\right|^{r-1} u_{t}(x, t-\tau)\right)$ and source $\left(|u|^{p-1} u\right)$ terms; hence, our work is the generalization of the above studies.

This work consists of five sections in addition to the introduction: Firstly, in Sect. 2, we recall some lemmas and assumptions. Then, in Section 3, we get the global existence of solutions. Moreover, in Section 4, we establish the decay results by using Nakao's tecnique. Finally, in Section 5, we obtain the blow-up of solutions for negative initial energy.

\section{Preliminaries}

In this part, we present some lemmas and assumptions for the proof of our result. Let $H^{m}(\Omega)$ denote the Sobolev space with the norm

$$
\|u\|_{H^{m}(\Omega)}=\left(\sum_{|\alpha| \leq m}\left\|D^{\alpha} u\right\|_{L^{2}(\Omega)}^{2}\right)^{1 / 2}
$$

$H_{0}^{m}(\Omega)$ denotes the closure in $H^{m}(\Omega)$ of $C_{0}^{\infty}(\Omega)$. For simplicity of notation, we denote by $\|\cdot\|_{p}$ the Lebesgue space $L^{p}(\Omega)$ norm, $\|\cdot\|$ denotes $L^{2}(\Omega)$ norm, and we write equivalent norm $\|\nabla \cdot\|$ instead of $H_{0}^{1}(\Omega)$ norm $\|\cdot\| H_{0}^{1}(\Omega)$. We denote by $C_{i}(i=1,2, \cdots, n)$ various positive constants which may be different at different occurrences.

Lemma 1 (see $[46,47]$ Sobolev-Poincarè inequality). If $2 \leq$ $p \leq\left(2 n /[n-2 m]^{+}\right)(2 \leq p<\infty$ if $n=2 m)$, then for some $C_{*}$,

$\|u\|_{p} \leq C_{*}\left\|(-\Delta)^{m / 2} u\right\|$ for $u \in H_{0}^{m}(\Omega)$

where we put $[\alpha]^{+}=\max \{0, \alpha\}, 1 /[\alpha]^{+}=\infty$ if $[\alpha]^{+}=0$.
Lemma 2 (see [48]). Let $\phi(t)$ be nonincreasing and nonnegative function defined on $[0, T], T>1$ and satisfies

$$
\phi^{1+\alpha}(t) \leq w_{0}(\phi(t)-\phi(t+1)), t \in[0, T]
$$

for $w_{0}$ is a positive constant, and $\alpha$ is a nonnegative constant. Then, we have for each $t \in[0, T]$,

$$
\begin{cases}\phi(t) \leq \phi(0) e^{-w_{1}[t-1]^{+}}, & \alpha=0, \\ \phi(t) \leq\left(\phi(0)^{-\alpha}+w_{0}^{-1} \alpha[t-1]^{+}\right)^{-1 / \alpha}, & \alpha=0,\end{cases}
$$

where $[t-1]^{+}=\max \{t-1,0\}$, and $w_{1}=\ln \left(w_{0} / w_{0}-1\right)$. We make the assumptions on parameters $r, p$, and $m$ as follows:

(A1)

$$
\left\{\begin{array}{l}
1<p<\infty, \quad n \leq 2 m, \\
1<p<\frac{n}{n-2 m}, n>2 m
\end{array}\right.
$$

$$
\left\{\begin{array}{l}
1<r<\infty, \quad n \leq 2 m, \\
1<r<\frac{n+2 m}{n-2 m}, \quad n>2 m .
\end{array}\right.
$$

\section{Global Existence}

In this part, we consider the global existence results of the problem (1). Firstly, we introduce the new function $z$ similar to the [7],

$$
z(x, k, t)=u_{t}(x, t-\tau k), x \in \Omega, k \in(0,1) .
$$

Thus, we have

$$
\tau z_{t}(x, k, t)+z_{k}(x, k, t)=0, \operatorname{in} \Omega \times(0,1) \times(0, \infty) .
$$

Hence, problem (1) can be transformed as follows:

$$
\left\{\begin{array}{lc}
u_{t t}+\left(\int_{\Omega}\left|A^{m / 2} u\right|^{2} d x\right)^{q} A^{m} u+\mu_{1}\left|u_{t}(x, t)\right|^{r-1} u_{t}(x, t)+\mu_{2}|z(x, 1, t)|^{r-1} z(x, 1, t)=|u|^{p-1} u & (x, t) \in \Omega \times(0, T) \\
\tau x_{t}(x, k, t)+z_{k}(x, k, t)=0 & \text { in } \Omega \times(0,1) \times(0, \infty) \\
z(x, k, 0)=f_{0}(x,-\tau k) & x \in \Omega, \\
u(x, 0)=u_{0}(x), u_{t}(x, 0)=u_{1}(x) & x \in \Omega, \\
\frac{\partial^{i} u}{\partial v^{i}}=0, i=0,1, \cdots, m-1 & x \in \partial \Omega .
\end{array}\right.
$$


We define the energy functional for any regular solution of (22) as follows:

$$
\begin{aligned}
E(t)= & \frac{1}{2}\left\|u_{t}\right\|^{2}+\frac{1}{2(q+1)}\left\|A^{m / 2} u\right\|^{2(q+1)}-\frac{1}{p+1}\|u\|_{p+1}^{p+1} \\
& +\frac{\varsigma}{r+1} \int_{\Omega} \int_{0}^{1} z^{r+1}(x, k, s) d k d x
\end{aligned}
$$

such that

$$
\tau r\left|\mu_{2}\right|<\varsigma<\tau\left((r+1) \mu_{1}-\left|\mu_{2}\right|\right) .
$$

Also, have

$$
\begin{aligned}
J(t)= & J(u(t))=\frac{1}{2(q+1)}\left\|A^{m / 2} u\right\|^{2(q+1)}-\frac{1}{p+1}\|u\|_{p+1}^{p+1} \\
& +\frac{\varsigma}{r+1} \int_{\Omega} \int_{0}^{1} z^{r+1}(x, k, s) d k d x
\end{aligned}
$$

$$
I(t)=I(u(t))=\left\|A^{m / 2} u\right\|^{2(q+1)}-\|u\|_{p+1}^{p+1}+\varsigma \int_{\Omega} \int_{0}^{1} z^{r+1}(x, k, s) d k d x .
$$

We easily see that

$$
E(t)=J(t)+\frac{1}{2}\left\|u_{t}\right\|^{2}
$$

Furthermore, we define

$$
\mathscr{W}=\left\{u: u \in H_{0}^{m}(\Omega) \cap H^{2 m}(\Omega), I(u)>0\right\} \cup\{0\} .
$$

Next, lemma gives that the energy functional $E(t)$ is a nonincreasing.

Lemma 3. Assume that $(u, z)$ is the solution of (22), then for $t \geq 0$,

$$
\begin{aligned}
E^{\prime}(t)= & -\left(\mu_{1}-\frac{\varsigma}{\tau(r-1)}-\frac{\mu_{2}}{r+1}\right)\left\|u_{t}(t)\right\|_{r+1}^{r+1} \\
& -\left(\frac{\varsigma}{\tau(r+1)}-\frac{\mu_{2} r}{r+1}\right) \int_{\Omega} z^{r+1}(x, 1, t) d x \leq 0
\end{aligned}
$$

Proof. We multiply the first equation in (22) by $u_{t}$, integrate over, and use integration by parts, and we obtain

$$
\begin{aligned}
\frac{d}{d t} & {\left[\frac{1}{2}\left\|u_{t}(t)\right\|^{2}+\frac{1}{2(q+1)}\left\|A^{m / 2} u\right\|^{2(q+1)}-\frac{1}{p+1}\|u\|_{p+1}^{p+1}\right] } \\
& +\mu_{1}\left\|u_{t}(t)\right\|_{r+1}^{r+1} \int_{\Omega} \mu_{2}|z(x, 1, t)|^{r-1} z(x, 1, t) u_{t}(x, t) d x=0 .
\end{aligned}
$$

Integrating (30) over $(0, t)$, we get

$$
\begin{aligned}
& {\left[\frac{1}{2}\left\|u_{t}(t)\right\|^{2}+\frac{1}{2(q+1)}\left\|A^{m / 2} u\right\|^{2(q+1)}-\frac{1}{p+1}\|u\|_{p+1}^{p+1}\right]} \\
& +\int_{0}^{t} \mu_{1}\left\|u_{s}(s)\right\|_{r+1}^{r+1} d s+\mu_{2} \int_{0}^{t} \int_{\Omega}|z(x, 1, s)|^{r-1} z(x, 1, s) u_{s}(x, s) d x d s \\
& \quad=\frac{1}{2}\left\|u_{1}\right\|^{2} .
\end{aligned}
$$

We multiply the second equation in (22) by $\varsigma|z|^{r-1} z$ and integrate the result over $\Omega \times(0,1) \times(0, t)$, and we get

$$
\begin{aligned}
& \frac{\varsigma}{r+1} \frac{d}{d t} \int_{0}^{t} \int_{\Omega} \int_{0}^{1}|z(x, k, t)|^{r-1}(x, k, t) z_{t}(x, k, t) d k d x d s \\
& =-\frac{\varsigma}{\tau(r+1)} \int_{0}^{t} \int_{\Omega} \int_{0}^{1} \frac{\partial}{\partial k}|z(x, k, t)|^{r+1} d k d x d s \\
& =-\frac{\varsigma}{\tau(r+1)} \int_{0}^{t} \int_{\Omega}\left[|z(x, 1, t)|^{r+1}-|z(x, 0, t)|^{r+1}\right] d x d s \\
& =-\frac{\varsigma}{\tau(r+1)} \int_{0}^{t} \int_{\Omega}|z(x, 1, t)|^{r+1} d x d s \\
& \quad+\frac{\varsigma}{\tau(r+1)} \int_{0}^{t}\left\|u_{t}(t)\right\|_{r+1}^{r+1} d s .
\end{aligned}
$$

By combining (31) and (32), we arrive at

$$
\begin{aligned}
E(t) & +\left(\mu_{1}-\frac{\varsigma}{\tau(r+1)}\right) \int_{0}^{t}\left\|u_{s}(s)\right\|_{r+1}^{r+1} d s \\
& +\frac{\varsigma}{\tau(r+1)} \int_{0}^{t} \int_{\Omega}|z(x, 1, s)|^{r+1} d x d s \\
& +\mu_{2} \int_{0}^{t} \int_{\Omega}|z(x, 1, s)|^{r-1} z(x, 1, s) u_{s}(x, s) d x d s=E(0) .
\end{aligned}
$$

Utilizing the Young inequality on the fourth term of the left hand side of (33), we conclude that

$$
\begin{aligned}
E(t) & +\left(\mu_{1}-\frac{\varsigma}{\tau(r+1)}-\frac{\mu_{2}}{r+1}\right) \int_{0}^{t}\left\|u_{s}(s)\right\|_{r+1}^{r+1} d s \\
& +\left(\frac{\varsigma}{\tau(r+1)}-\frac{\mu_{2} r}{r+1}\right) \int_{0}^{t} \int_{\Omega}|z(x, 1, s)|^{r+1} d x d s=E(0)
\end{aligned}
$$

Deriving the (34), we have the desired result. Hence, the proof is completed.

Remark 4. From the condition (24), we obtain

$c_{1}=\left(\mu_{1}-\frac{\varsigma}{\tau(r+1)}-\frac{\mu_{2}}{r+1}\right)>0, c_{2}=\left(\frac{\varsigma}{\tau(r+1)}-\frac{\mu_{2} r}{r+1}\right)>0$. 
Lemma 5. Assume that (19) and $p>2 q+1$ hold. Let $u_{0} \in \mathscr{W}$ and $u_{1} \in H_{0}^{m}(\Omega)$, such that

$$
\beta=C_{*}\left(\frac{2(q+1)(p+1)}{p-2 q-1} E(0)\right)^{p-2 q-1 / 2(q+1)}<1,
$$

then $u \in \mathscr{W}$ for each $t \geq 0$.

Proof. It follows the continuity of $u(t)$, since $I(0)>0$, such that

$$
I(t)>0
$$

for some interval near $t=0$. Assume that $T_{m}>0$ is a maximal time, when (26) holds on $\left[0, T_{m}\right]$.

By (25) and (26), we obtain

$$
\begin{aligned}
J(t)= & \frac{1}{p+1} I(t)+\frac{p-2 q-1}{2(q+1)(p+1)}\left\|A^{m / 2} u\right\|^{2(q+1)} \\
& +\frac{(p-r)}{(r+1)(p+1)}\left(\varsigma \int_{\Omega} \int_{0}^{1} z^{r+1}(x, k, t) d k d x\right) \\
\geq & \frac{p-2 q-1}{2(q+1)(p+1)}\left\|A^{m / 2} u\right\|^{2(q+1)} .
\end{aligned}
$$

From (23), (38), and Lemma 3, we have

$$
\begin{aligned}
\left\|A^{m / 2} u\right\|^{2(q+1)} & \leq \frac{2(q+1)(p+1)}{p-2 q-1} J(t) \leq \frac{2(q+1)(p+1)}{p-2 q-1} E(t) \\
& \leq \frac{2(q+1)(p+1)}{p-2 q-1} E(0) .
\end{aligned}
$$

Using Lemma 1 and (39), we get

$$
\begin{aligned}
\|u\|_{p+1}^{p+1} & \leq C_{*}\left\|A^{m / 2} u\right\|^{p+1}=C_{*}\left\|A^{m / 2} u\right\|^{p-2 q-1}\left\|A^{m / 2} u\right\|^{2(q+1)} \\
& \leq C_{*}\left(\frac{2(q+1)(p+1)}{p-2 q-1} E(0)\right)^{p-2 q-1 / 2(q+1)}\left\|A^{m / 2} u\right\|^{2(q+1)} \\
& =\beta\left\|A^{m / 2} u\right\|^{2(q+1)}<\left\|A^{m / 2} u\right\|^{2(q+1)} \text { on } \mathrm{t} \in\left[0, T_{m}\right] .
\end{aligned}
$$

Thus, from (26), we arrive at $I(t)>0$ for all $t \in\left[0, T_{m}\right]$. $T_{m}$ is extended to $T$, by repeating the procedure. Hence, the proof is completed.

Lemma 6. Suppose that the assumptions of Lemma 5 hold. Then, there exists $\eta_{1}=1-\beta$, such that

$$
\|u\|_{p+1}^{p+1} \leq\left(1-\eta_{1}\right)\left\|A^{m / 2} u\right\|^{2(q+1)} .
$$

Proof. By (40), we obtain

$$
\|u\|_{p+1}^{p+1} \leq \beta\left\|A^{m / 2} u\right\|^{2(q+1)} .
$$

Let $\eta_{1}=1-\beta$; therefore, we obtain the result.
Remark 7. By Lemma 6, we conclude that

$$
\left\|A^{m / 2} u\right\|^{2(q+1)} \leq \frac{1}{\eta_{1}} I(t) .
$$

Theorem 8. Assume that the assumptions (A2), $\mu_{2}<\mu_{1}$, and $p>2 q+1$ hold. Let $u_{0} \in \mathscr{W}$ satisfying (36) and $f_{0} \in L^{2}(\Omega \times($ $0,1)$ ) be given. Then, the solution of problem (22) is global.

Proof. It is sufficient to show that $\left\|u_{t}\right\|^{2}+\left\|A^{m / 2} u\right\|^{2(q+1)}$ is bounded independently of $t$. To obtain this, by using (23) and (26), we have

$$
\begin{aligned}
E(0) \geq & E(t)=\frac{1}{2}\left\|u_{t}\right\|^{2}+\frac{1}{2(q+1)}\left\|A^{m / 2} u\right\|^{2(q+1)}-\frac{1}{p+1}\|u\|_{p+1}^{p+1} \\
& +\frac{\varsigma}{r+1} \int_{\Omega} \int_{0}^{1} z^{r+1}(x, k, x) d k d x=\frac{1}{2}\left\|u_{t}\right\|^{2} \\
& +\frac{p-2 q-1}{2(q+1)(p+1)}\left\|A^{m / 2} u\right\|^{2(q+1)} \\
& +\frac{(p-r)}{(r+1)(p+1)}\left(\varsigma \int_{\Omega} \int_{0}^{1} z^{r+1}(x, k, t) d k d x\right) \\
& +\frac{1}{p+1} I(t) \geq \frac{1}{2}\left\|u_{t}\right\|^{2}+\frac{p-2 q-1}{2(q+1)(p+1)}\left\|A^{m / 2} u\right\|^{2(q+1)}
\end{aligned}
$$

since $I(t) \geq 0$. Thus,

$$
\left\|u_{t}\right\|^{2}+\left\|A^{m / 2} u\right\|^{2(q+1)} \leq C E(0)
$$

where $C=\max \{2,(2(q+1)(p+1) / p-2 q-1)\}$. Therefore, we obtain the global existence of solutions. Therefore, we completed the proof.

\section{Decay of Solution}

In this part, we obtain the decay of solutions of the problem (22) by using Nakao's technique.

Theorem 9. Assume that the assumption (A2) and (36) hold. Let $u_{0} \in \mathscr{W}, f_{0} \in L^{2}(\Omega \times(0,1))$, be given. Hence, we have following decay estimates:

$$
E(t) \leq \begin{cases}E(0) e^{-w_{1}[t-1]^{+}}, & \text {if } r=1, \\ \left(E(0)^{-\alpha}+C_{7}^{-1} \alpha[t-1]^{+}\right)^{-1 / \alpha}, & \text { if } r>1,\end{cases}
$$

where $w_{1}, \alpha$ and $C_{7}$ are positive constants which will be defined later.

Proof. We integrate (29) over $[t, t+1], t>0$, to get

$$
E(t)-E(t+1)=[D(t)]^{r+1}
$$


where

$$
[D(t)]^{r+1}=c_{1} \int_{t}^{t+1}\left\|u_{t}\right\|_{r+1}^{r+1} d s+c_{2} \int_{t}^{t+1} \int_{\Omega} z^{r+1}(x, 1, s) d x d s
$$

From (48) and Hölder inequality, we see that

$$
\int_{t}^{t+1} \int_{\Omega}\left|u_{t}\right|^{2} d x d t+\int_{t}^{t+1} \int_{\Omega}|z(x, 1, s)|^{2} d x d s \leq x(\Omega)[D(t)]^{2},
$$

where $c(\Omega)=\operatorname{vol}(\Omega)$. Therefore, by (49), there exists $t_{1}$ $\in[t, t+1 / 4]$ and $t_{2} \in[t+3 / 4, t+1]$, so that

$$
\left\|u_{t}\left(t_{i}\right)\right\|^{2}+\left\|z\left(x, 1, t_{i}\right)\right\|^{2} \leq c(\Omega)[D(t)]^{2}, i=1,2 .
$$

We multiply the first equation in (22) by $u$ and integrate over $\Omega \times\left[t_{1}, t_{2}\right]$. Use integration by parts, Hölder's inequality, adding, and subtracting the term $\int_{t_{1}}^{t_{2}} \int_{\Omega} \int_{0}^{1} \varsigma z^{r+1}(x, k, t) d k$ $d x d t$, we have

$$
\begin{aligned}
\int_{t_{1}}^{t_{2}} I(t) d t \leq & \left\|u_{t}\left(t_{1}\right)\right\|_{2}\left\|u\left(t_{1}\right)\right\|_{2}+\left\|u_{t}\left(t_{2}\right)\right\|_{2}\left\|u\left(t_{2}\right)\right\|_{2} \\
& +\int_{t_{1}}^{t_{2}}\left\|u_{t}\right\|^{2} d t+\int_{t_{1}}^{t_{2}} \int_{0}^{1} \varsigma \int_{\Omega} z^{r+1}(x, k, t) d x d k d t \\
& -\mu_{1} \int_{t_{1}}^{t_{2}} \int_{\Omega}\left|u_{t}\right|^{r-1}\left|u_{t}\right| u d x d t \\
& -\mu_{2} \int_{t_{1}}^{t_{2}} \int_{\Omega}|z(x, 1, t)|^{r-1} z(x, 1, t) u d x d t .
\end{aligned}
$$

Now, we estimate the right hand side for (51).

From (39), (50), and Lemma 1, we obtain

$$
\left\|u_{t}\left(t_{i}\right)\right\|_{2}\left\|u\left(t_{i}\right)\right\|_{2} \leq C_{1} D(t) \sup _{t_{1} \leq s \leq t_{2}} E^{1 / 2}(s),
$$

where $C_{1}=2 C_{*}((2(q+1)(p+1) / p-2 q-1) E(0))^{1 / 2(q+1)}$.

By using (32) that

$$
\begin{gathered}
\int_{t_{1}}^{t_{2}} \int_{0}^{1} \int_{\Omega} z^{r+1}(x, k, t) d x d k d t \leq \frac{1}{2 \tau} \int_{t_{1}}^{t_{2}} \int_{t_{1}}^{t_{2}}\left\|u_{t}(s)\right\|_{r+1}^{r+1} d s d v \\
\quad \leq\left(\int_{t_{1}}^{t_{2}} d v\right)\left(\int_{t_{1}}^{t_{2}}\left\|u_{t}(s)\right\|_{r+1}^{r+1} d s\right) \leq\left(t_{2}-t_{1}\right)[D(t)]^{r+1} .
\end{gathered}
$$

Utilizing Hölder inequality, we get

$$
\int_{t_{1}}^{t_{2}} \int_{\Omega}\left|u_{t}\right|^{r-1}\left|u_{t}\right| u d x d t \leq \int_{t_{1}}^{t_{2}}\left\|u_{t}(t)\right\|_{r+1}^{r}\|u(t)\|_{r+1} .
$$

Utilizing the Sobolev-Poincare inequality and (39), we have

$$
\begin{aligned}
& \left|\int_{t_{1}}^{t_{2}}\left\|u_{t}(t)\right\|_{r+1}^{r}\|u(t)\|_{r+1} d t\right| \leq C_{*} \int_{t_{1}}^{t_{2}}\left\|u_{t}(t)\right\|_{r+1}^{r}\left\|A^{m / 2} u\right\| d t \\
& \quad \leq C_{*}\left(\frac{2(q+1)(p+1)}{p-2 q-1} E(0)\right)^{1 / 2(q+1)} \int_{t_{1}}^{t_{2}}\left\|u_{t}(t)\right\|_{r+1}^{r} E^{1 / 2}(s) d t \\
& \quad \leq C_{*}\left(\frac{2(q+1)(p+1)}{p-2 q-1} E(0)\right)^{1 / 2(q+1)} \sup _{t_{1} \leq s \leq t_{2}} E^{1 / 2}(s) \int_{t_{1}}^{t_{2}}\left\|u_{t}(t)\right\|_{r+1}^{r} d t \\
& \quad \leq C_{*}\left(\frac{2(q+1)(p+1)}{p-2 q-1} E(0)\right)^{1 / 2(q+1)} \sup _{t_{1}, \leq s \leq t_{2}} E^{1 / 2}(s)[D(t)]^{r} .
\end{aligned}
$$

Also, we obtain

$$
\begin{aligned}
& \int_{t_{1}}^{t_{2}} \int_{\Omega}|z(x, 1, t)|^{r-1} z(x, 1, t) u d x d t \leq C_{*} \int_{t_{1}}^{t_{2}}\|z(x, 1, t)\|_{r+1}^{r}\|u\|_{r+1} d t \\
& \quad \leq C_{*}\left(\frac{2(q+1)(p+1)}{p-2 q-1} E(0)\right)^{1 / 2(q+1)} \int_{t_{1}}^{t_{2}}\|z(x, 1, t)\|_{r+1}^{r} E^{1 / 2}(s) d t \\
& \quad \leq C_{*}\left(\frac{2(q+1)(p+1)}{p-2 q-1} E(0)\right)^{1 / 2(q+1)} \sup _{t_{1} \leq s \leq t_{2}} E^{1 / 2}(s) \int_{t_{1}}^{t_{2}}\|z(x, 1, t)\|_{r+1}^{r} d t \\
& \quad \leq C_{*}\left(\frac{2(q+1)(p+1)}{p-2 q-1} E(0)\right)^{1 / 2(q+1)} \sup _{t_{1} \leq s \leq t_{2}} E^{1 / 2}(s)[D(t)]^{r} .
\end{aligned}
$$

Then, from (51)-(56), we get

$$
\begin{aligned}
\int_{t_{1}}^{t_{2}} I(t) d t \leq & C_{2}\left[\sup _{t_{1} \leq s \leq t_{2}} E^{1 / 2}(s) D(t)+[D(t)]^{2}+\left(t_{2}-t_{1}\right)[D(t)]^{r+1}\right. \\
& \left.+2 C_{*}\left(\frac{2(q+1)(p+1)}{p-2 q-1} E(0)\right)^{1 / 2(q+1)} \sup _{t_{1} \leq s \leq t_{2}} E^{1 / 2}(s)[D(t)]^{r}\right] .
\end{aligned}
$$

Moreover, by (23), (26), and Remark 7, we have

$$
E(t) \leq \frac{1}{2}\left\|u_{t}\right\|^{2}+C_{3} I(t)
$$

where $C_{3}=\left(1 / \eta_{1}\right)(p-2 q-1 / 2(q+1)(p+1))+(1 / p+1)$

Integrating (58) over $\left[t_{1}, t_{2}\right]$, we get

$$
\int_{t_{1}}^{t_{2}} E(t) d t \leq \frac{1}{2} \int_{t_{1}}^{t_{2}}\left\|u_{t}\right\|^{2} d t+C_{3} \int_{t_{1}}^{t_{2}} I(t) d t
$$


Hence, from (57) and (59), we obtain

$$
\begin{aligned}
\int_{t_{1}}^{t_{2}} E(t) d t \leq & \frac{1}{2} C[D(t)]^{2}+C_{3} C_{2}\left[\sup _{t_{1} \leq s \leq t_{2}} E^{1 / 2}(s) D(t)+[D(t)]^{2}\right. \\
& +\left(t_{2}-t_{1}\right)[D(t)]^{r+1}+2 C_{*} \\
& \left.\cdot\left(\frac{2(q+1)(p+1)}{p-2 q-1} E(0)\right)^{1 / 2(q+1)} \sup _{t_{1} \leq s \leq t_{2}} E^{1 / 2}(s)[D(t)]^{r}\right] .
\end{aligned}
$$

Integrating $(d / d t) E(t)$ over $[t, t 2]$, we conclude that

$$
\begin{aligned}
E(t)= & E\left(t_{2}\right)+\int_{t}^{t_{2}}\left(\mu_{1}-\frac{\varsigma}{\tau(r+1)}-\frac{\mu_{2}}{r+1}\right)\left\|u_{s}(s)\right\|_{r+1}^{r+1} d s \\
& +\int_{t}^{t_{2}}\left(\frac{\varsigma}{\tau(r+1)}-\frac{\mu_{2} r}{r+1}\right) \int_{\Omega}|z(x, 1, s)|^{r+1} d x d s .
\end{aligned}
$$

Thus, since $t_{2}-t_{1} \geq 1 / 2$, we arrive at

$$
\int_{t_{1}}^{t_{2}} E(t) d t \geq\left(t_{2}-t_{1}\right) E\left(t_{2}\right) \geq \frac{1}{2} E\left(t_{2}\right) .
$$

Hence,

$$
E\left(t_{2}\right) \leq 2 \int_{t_{1}}^{t_{2}} E(t) d t
$$

As a result, from (47), (60), (61), (63), and since $t_{1}, t_{2}$ $\in[t, t+1]$, we get

$$
\begin{aligned}
E(t) \leq & 2 \int_{t_{1}}^{t_{2}} E(t) d t+\int_{t}^{t+1}\left(\mu_{1}-\frac{\varsigma}{\tau(r+1)}-\frac{\mu_{2}}{r+1}\right)\left\|u_{s}(s)\right\|_{r+1}^{r+1} d s \\
& +\int_{t}^{t+1}\left(\frac{\varsigma}{\tau(r+1)}-\frac{\mu_{2} r}{r+1}\right) \int_{\Omega}|z(x, 1, s)|^{r+1} d x d s \\
= & 2 \int_{t_{1}}^{t_{2}} E(t) d t+[D(t)]^{r+1} .
\end{aligned}
$$

Then, from (60), we have

$$
\begin{aligned}
E(t) \leq & \left(\frac{1}{2} C+C_{3} C_{2}\right)[D(t)]^{2}+C_{3} C_{2}[D(t)]^{r+1} \\
& +C_{4}\left[D(t)+[D(t)]^{r}\right] E^{1 / 2}(t) .
\end{aligned}
$$

Thus, utilizing Young inequality, we have

$$
E(t) \leq C_{5}\left[[D(t)]^{2}+[D(t)]^{r+1}+[d(t)]^{2 r}\right]
$$

Hence, we have the decay estimates as follows:
Case 1. If $r=1$, by (66), we obtain

$$
E(t) \leq 3 C_{5}[D(t)]^{2}=3 C_{5}[E(t)-E(t-1)] .
$$

Utilizing Lemma 2, we have

$$
E(t) \leq E(0) e^{-w_{1}[t-1]^{+}}
$$

where $w_{1}=\ln \left(3 C_{5} / 3 C_{5}-1\right)$.

Case 2. If $r>1$, by (66), we have

$$
E(t) \leq C_{5}[D(t)]^{2}\left(1+[D(t)]^{r-1}+[D(t)]^{2 r-2}\right) .
$$

Then, by (47), since $E(t) \leq E(0), \forall t \geq 0$, we see that

$$
\begin{aligned}
E(t) & \leq C_{5}\left(1+E^{r-1 / r+1}(0)+E^{2(r-1) / r+1}(0)\right)[D(t)]^{2} \\
& \leq C_{6}[D(t)]^{2}, t \geq 0 .
\end{aligned}
$$

Then, we get

$$
E(t)^{r+1 / 2} \leq C_{7}[D(t)]^{r+1} \leq C_{7}(E(t)-E(t+1)) .
$$

Therefore, by (71) and Lemma 2, we obtain

$$
E(t) \leq\left(E(0)^{-\alpha}+C_{7}^{-1} \alpha[t-1]^{+}\right)^{-1 / \alpha}
$$

Thus, we completed the proof of Theorem 9.

\section{Blow-Up of Solution}

In this part, we get the blow-up of solutions for negative initial energy, in the case $r>1$.

Theorem 10. Let $\left(u_{0}, u_{1}\right) \in\left(H^{2 m}(\Omega) \cap H_{0}^{m}(\Omega)\right) \times H_{0}^{m}(\Omega)$ and $f_{0} \in L^{2}(\Omega \times(0,1))$ be given. Assume that $p>\max \{2, r$, $2 q+1\}$ and the assumptions (A1)-(A2) hold. Then, the solution of (22) blows up in a finite time with $E(0)<0$.

Proof. Setting

$$
H(t)=-E(t),
$$

from Lemma 3, we obtain

$$
\begin{aligned}
H^{\prime}(t) \geq & -\left(\mu_{1}-\frac{\varsigma}{\tau(r+1)}-\frac{\mu_{2}}{r+1}\right)\left\|u_{t}(t)\right\|_{r+1}^{r+1} \\
& -\left(\frac{\varsigma}{\tau(r+1)}-\frac{\mu_{2} r}{r+1}\right) \int_{\Omega} z^{r+1}(x, 1, t) d x .
\end{aligned}
$$

Thus,

$$
0<H(0) \leq H(t) \leq \frac{1}{p+1}\|u\|_{p+1}^{p+1}, t>0
$$


Let

$$
M(t)=\|u\|_{2}^{2} .
$$

Differentiating (76) twice, we get

$$
\begin{gathered}
M^{\prime}(t)=2 \int_{\Omega} u_{t} u d x, \\
M^{\prime \prime}(t)=2\left\|u_{t}\right\|^{2}+2 \int_{\Omega} u_{t t} u d x .
\end{gathered}
$$

Using the first equation in (22), to have

$$
\begin{aligned}
M^{\prime \prime}(t)= & 2\left\|u_{t}\right\|^{2}-2\left\|A^{\frac{m}{2}} u\right\|_{2}^{2(q+1)}-2 \mu_{1} \int_{\Omega}\left|u_{t}(x, t)\right|^{r-1} u u_{t}(x, t) d x \\
& -2 \mu_{2} \int_{\Omega}|z(x, 1, t)|^{r-1} u z(x, 1, t) d x+2\|u\|_{p+1}^{p+1},
\end{aligned}
$$

we add and subtract the term $2(p+1) H(t)$, and then (78) becomes the form

$$
\begin{aligned}
M^{\prime \prime}(t) \geq & (p+3)\left\|u_{t}\right\|^{2}+2(p+1) H(t) \\
& +\left(\frac{p+1}{q+1}-2\right)\left\|A^{\frac{m}{2}} u\right\|_{2}^{2(q+1)} \\
& -2 \mu_{1} \int_{\Omega}\left|u_{t}(x, t)\right|^{r-1} u u_{t}(x, t) d x \\
& -2 \mu_{2} \int_{\Omega}|z(x, 1, t)|^{r-1} u z(x, 1, t) d x \\
& +\frac{2 \varsigma(p+1)}{r+1} \int_{\Omega} \int_{0}^{1} z^{r+1}(x, k, s) d k d x .
\end{aligned}
$$

Now, we define

$$
L(t)=H(t)^{1-\kappa}+2 \epsilon M^{\prime}(t)
$$

Differentiating (80), we obtain

$$
L^{\prime}(t)=(1-\kappa) H(t)^{-\kappa} H^{\prime}(t)+2 \varepsilon M^{\prime \prime}(t) .
$$

Replacing (79) in (81), we arrive at

$$
\begin{aligned}
L^{\prime}(t) \geq & (1-\kappa) H(t)^{-\kappa} H^{\prime}(t) 2 \varepsilon(p+3)\left\|\mu_{t}\right\|^{2} \\
& +4 \varepsilon(p+1) H(t)+2 \varepsilon\left(\frac{p+1}{q+1}-2\right)\left\|A^{\frac{m}{2}} \mu\right\|_{2}^{2(q+1)} \\
& -4 \varepsilon \mu_{1} \int_{\Omega}\left|\mu_{t}(x, t)\right|^{r-1} u u_{t}(x, t) d x \\
& -4 \varepsilon \mu_{2} \int_{\Omega}|z(x, 1, t)|^{r-1} u z(x, 1, t) d x \\
& +\frac{4 \varepsilon \varsigma(p+1)}{r+1} \int_{\Omega} \int_{0}^{1} z^{r+1}(x, k, s) d k d x .
\end{aligned}
$$

From (75) and utilizing Hölder inequality, we get

$$
\begin{aligned}
& \left.\left|\int_{\Omega}\right| u_{t}\right) x,\left.t\right|^{r-1} u u_{t}(x, t) d x \mid \leq\left\|u_{t}\right\|_{r+1}^{r}\|u\|_{r+1} \\
& \quad \leq c_{1}\|u\|_{r+1}^{r+1 / p+1}\|u\|_{r+1}^{1-r+1 / p+1}\|u\|_{r+1}^{r} \\
& \quad \leq c_{2}\|u\|_{r+1}^{r+1 / p+1} H(t)^{\frac{1}{p+1}-\frac{r+1}{(p+1)^{2}}}\left\|u_{t}\right\|_{r+1}^{r} .
\end{aligned}
$$

From Young's inequality and (74), we have

$$
\begin{aligned}
& \left.\left|\int_{\Omega}\right| u_{t}(x, t)\right|^{r-1} u u_{t}(x, t) d x \mid \\
& \quad \leq c_{3}\left(\rho^{1 / 1+p}\|\mu\|_{r+1}^{r+1} H(0)^{-\bar{k}}+\rho^{-r^{\prime}} H(0)^{k-\bar{k}} H^{\prime}(t) H(t)^{-k}\right),
\end{aligned}
$$

where $\bar{k}=1 / p+1-r+1 /(p+1)^{2}>0, \rho>0, r^{\prime}=r+1 / r$, letting $0<k<\bar{k}$. In a similar way, we obtain

$$
\begin{aligned}
& \left.\left|\int_{\Omega} u\right| z(x, 1, t)\right|^{r-1} z(x, 1, t) d x \mid \\
& \quad \leq c_{3}\left(\rho^{\frac{1}{1+p}}\|\mu\|_{r+1}^{r+1} H(0)^{-\bar{k}}+\rho^{-r^{\prime}} H(0)^{k-\bar{k}} H^{\prime}(t) H(t)^{-k}\right) .
\end{aligned}
$$

By using (82), (84), and (85), to have

$$
\begin{aligned}
L^{\prime}(t) \geq & {\left[(1-\kappa)-4 \varepsilon\left(\mu_{1}+\mu_{2}\right) H^{k-\bar{k}}(0) \rho^{-r^{\prime}}\right] H(t)^{-\kappa} H^{\prime}(t) } \\
& -4 \varepsilon\left(\mu_{1}+\mu_{2}\right) H(0)^{-\bar{k}} \rho^{1 / 1+p}\|u\|_{r+1}^{r+1}+2 \varepsilon(p+3)\left\|u_{t}\right\|^{2} \\
& +4 \varepsilon(p+1) H(t)+2 \varepsilon\left(\frac{p+1}{q+1}-2\right)\left\|A^{m / 2} \mu\right\|_{2}^{2(q+1)} \\
& +\frac{4 \varepsilon \varsigma(p+1)}{r+1} \int_{\Omega} \int_{0}^{1} z^{r+1}(x, k, s) d k d x,
\end{aligned}
$$

for $\varepsilon$ sufficiently small, we obtain

$$
\left[(1-\kappa)-4 \varepsilon\left(\mu_{1}+\mu_{2}\right) H^{k-\bar{k}}(0) \rho^{-r^{\prime}}\right] \geq 0 .
$$

Setting $s=r+1 \leq p+1$ such that

$$
\|\mu\|_{r+1}^{s} \leq c_{1}\left(\left\|A^{m / 2} \mu\right\|+\|\mu\|_{p+1}^{p+1}\right),
$$

where $c=4\left(\mu_{1}+\mu_{2}\right) H(0)^{-\bar{k}} \rho^{1 / p+1} c_{1}$ and taking $(p+1 / q+1$ $-2)>c$. Thus, we have

$$
\begin{aligned}
L^{\prime}(t) \geq & 2 \varepsilon\left(\frac{p+1}{q+1}-2-c\right)\left\|A^{m / 2} u\right\|_{2}^{2(q+1)} \\
& -\varepsilon c\|\mu\|_{p+1}^{p+1}+4 \varepsilon(p+1) H(t)+2 \varepsilon(p+3)\|\mu\|^{2} \\
& +4 \varepsilon \frac{\zeta(p+1)}{r+1} \int_{\Omega} \int_{0}^{1} z^{r+1}(x, k, s) d k d x .
\end{aligned}
$$


By using the notations $a_{1}=2(p+1 / q+1-2-c), a_{2}=c$, $a_{3}=4(p+1)$, and $a_{4}=2(p+3)$, (89) takes the form

$L^{\prime}(t) \geq a_{1} \varepsilon\left\|A^{m / 2} \mu\right\|_{2}^{2(q+1)}-\varepsilon a_{2}\|\mu\|_{p+1}^{p+1}+\varepsilon a_{3} H(t)+\varepsilon a_{4}\left\|\mu_{t}\right\|^{2}$.

Similarly to the approach of Messaoudi [49], we assume that $p=2 a_{5}+\left(p-2 a_{5}\right)$, where $a_{5}<\min \left(a_{1}, a_{2}, a_{3}, a_{4}\right)$, and then (90) becomes the form

$$
\begin{aligned}
L^{\prime}(t) \geq & \left(a_{1}-a_{5}\right) \varepsilon\left\|A^{m / 2} \mu\right\|_{2}^{2(q+1)}+\varepsilon\left(a_{5}-a_{2}\right)\|\mu\|_{p+1}^{p+1} \\
& +\varepsilon\left(a_{3}-a_{5}\right) H(t)+\varepsilon\left(a_{4}-a_{5}\right)\left\|\mu_{t}\right\|^{2} .
\end{aligned}
$$

Then,

$$
L^{\prime}(t) \geq \delta \varepsilon\left[\left\|A^{\frac{m}{2}} u\right\|_{2}^{2(q+1)}+\left\|\mu_{t}\right\|_{p+1}^{p+1}+H(t)+\left\|u_{t}\right\|^{2}\right] .
$$

We conclude that

$$
L^{\prime}(t) \geq \delta \varepsilon\left[\|u\|_{p+1}^{p+1}+H(t)+\left\|u_{t}\right\|^{2}\right]
$$

where $\delta>0$ is the minimum of the coefficients of $\|u\|_{p+1}^{p+1}$, $H(t),\left\|u_{t}\right\|^{2}$. Pick out $\varepsilon$ such that

$$
L(0)=H^{1-\kappa}(0)+2 \varepsilon \int_{\Omega} u_{1} u_{0} d x>0 .
$$

As a result, we getsetting $\Phi=1 / 1-k$, and since $k<\bar{k}<1$, we see that $1<\emptyset<1 / 1-\bar{k}$. Set

$$
L(t)=H(t)^{1-\kappa}+2 \varepsilon \int_{\Omega} u u_{t} d x .
$$

Then,

$$
\begin{aligned}
L(t)= & H(t)^{1 / \omega}+2 \varepsilon \int_{\Omega} u u_{t} d x \leq H(t)^{1 / \omega} \\
& +2 \varepsilon \int_{\Omega} u u_{t} d x+2 E_{1}\left(\|u\|_{p+1}\right)^{p+1 / \omega}
\end{aligned}
$$

Utilizing Young, Hölder's inequalities, and (96), we conclude that

$$
\begin{aligned}
& L(t)^{\Phi} \leq\left[H(t)^{1 / \omega}+2 \varepsilon \int_{\Omega} u u_{t} d x+2 E_{1}\left(\|u\|_{p+1}\right)^{p+1 / \omega}\right]^{\Phi} \\
& \leq 2^{\omega-1}\left[H(t)+\left(2 \varepsilon \int_{\Omega} u u_{t} d x+2 E_{1}\left(\|u\|_{p+1}\right)^{p+1 / \infty}\right)^{\infty}\right] \\
& \leq 2^{\omega-1}\left[H(t)+2^{\omega-1}\left(\left(2 \varepsilon \int_{\Omega} u u_{t} d x\right)^{\Phi}+2 E_{1}\|u\|_{p+1}^{p+1}\right)\right] \\
& \leq 2^{\omega-1}\left[H(t)+2^{\omega-1}\left(\beta^{\Phi}\left\|u_{t}\right\|_{2}^{\Phi}\|u\|_{2}^{\Phi}+2 E_{1}\|u\|_{p+1}^{p+1}\right)\right] \\
& \leq 2^{\omega-1}\left[H(t)+2^{\omega-1}\left(\beta^{\Phi}\left\|u_{t}\right\|_{2}^{\infty}\|u\|_{2}^{\infty}\right)+2^{\omega-1}\left(2 E_{1}\|u\|_{p+1}^{p+1}\right)\right] \\
& \leq c_{2}[H(t)+]\left\|u_{t}\right\|_{2}^{\Phi}\|u\|_{2}^{\Phi}+\|u\|_{p+1}^{p+1} \text {, }
\end{aligned}
$$

where $c_{2}=\max \left\{2^{\omega-1}, \beta^{\infty}\right\}$. Furthermore, for $p>1$, utilizing Hölder and Young inequalities, we obtain

$$
\begin{gathered}
\left\|u_{t}\right\|_{2}^{\infty}\|u\|_{2}^{\infty} \leq c_{3}\left\|u_{t}\right\|_{2}^{\infty}\|u\|_{p+1}^{\infty} \leq c_{4}\left(\left\|u_{t}\right\|_{2}^{2}+\|u\|_{p+1}^{2(1-k) / 1-2 k}\right), \\
\|u\|_{p+1}^{2(1-k) / 1-2 k}=\|u\|_{p+1}^{p+1}\|u\|_{p+1}^{2(1-k) / 1-2 k-(p+1)} \\
\leq c_{5} H(0)^{2(1-k) /(1-2 k)(p+1)-1}\|u\|_{p+1}^{p+1} .
\end{gathered}
$$

Then, (97) becomes the form

$$
L(t)^{\Phi} \leq c_{6}\left(H(t)+\left\|u_{t}\right\|^{2}+\|u\|_{p+1}^{p+1}\right) .
$$

By combining (93) and (99), we conclude that

$$
L^{\prime}(t) \leq c_{7} L(t)^{\oplus}, c_{7}>0, \omega>1 .
$$

Therefore, a simple integration over $(0, t)$, we have the desired result. Hence, we completed the proof.

\section{Conclusions}

Time delays often appear in many various problems, such as, thermal, economic phenomena, biological, chemical, and physical. Recently, the partial differential equations with time delay have become an active area (see $[2,3]$ and references therein). In recent years, there has been published much work concerning the wave equation with constant delay or time-varying delay. However, to the best of our knowledge, there were no global existence, decay, and blow-up results for the higher-order Kirchhoff-type equation with delay term. Firstly, we have been obtained the global existence result. Later, we have been established the decay results by using Nakao's technique. Finally, we have proved the blow-up of solutions with negative initial energy for the problem (1) under the sufficient conditions in a bounded domain. In the next work, we will extend our current study to more general case of the problem (1). 


\section{Data Availability}

No data were used to support the study.

\section{Conflicts of Interest}

The authors declare that they do not have any conflicts of interest.

\section{References}

[1] G. Kirchho, Vorlesungen ber Mechanik, Teubner, Leipzig, 3rd edition, 1883.

[2] C. Abdallah, P. Dorato, J. Benitez-Read, and R. Byrne, Delayed positive feedback can stabilize oscillatory system, ACC, San Francisco, 1993.

[3] I. H. Suh and Z. Bien, "Use of time-delay actions in the controller design," IEEE Transactions on Automatic Control, vol. 25, no. 3, pp. 600-603, 1980.

[4] R. Datko, J. Lagnese, and M. P. Polis, "An example on the effect of time delays in boundary feedback stabilization of wave equations," SIAM Journal on Control and Optimization, vol. 24, no. 1, pp. 152-156, 1986.

[5] M. Kani and S. A. Messaoudi, "A blow-up result in a nonlinear wave equation with delay," The Mediterranean Journal of Mathematics, vol. 13, pp. 237-247, 2016.

[6] S. Nicaise and C. Pignotti, "Stability and instability results of the wave equation with a delay term in the boundary or internal feedbacks," SIAM Journal on Control and Optimization, vol. 45, no. 5, pp. 1561-1585, 2006.

[7] S. Nicaise and C. Pignotti, "Stabilization of the wave equation with boundary or internal distributed delay," Diff. Int. Equ., vol. 21, pp. 935-958, 2008.

[8] C. Q. Xu, S. P. Yung, and L. K. Li, "Stabilization of wave systems with input delay in the boundary control," ESAIM: Control, Optimisation and Calculus of Variations, vol. 12, no. 4, pp. 770-785, 2006.

[9] S. Nicaise, J. Valein, and E. Fridman, "Stabilization of the heat and the wave equations with boundary time-varying delays," DCDIS-S, vol. 2, no. 3, pp. 559-581, 2009.

[10] M. Kirane and B. Said-Houari, "Existence and asymptotic stability of a viscoelastic wave equation with a delay," Zeitschrift für Angewandte Mathematik und Physik, vol. 62, no. 6, pp. 1065-1082, 2011.

[11] F. Z. Mahdi, M. Ferhat, and A. Hakem, "Blow up and asymptotic behavior for a system of viscoelastic wave equations of kirchhoff-type with a delay term," ATNAA, vol. 2, no. 3, pp. 146-167, 2018.

[12] E. Pişkin and H. Yüksekkaya, "Nonexistence of Solutions of a Delayed Wave Equation with Variable-Exponents," C-POST, vol. 3, no. 1, pp. 97-101, 2020.

[13] E. Pişkin and H. Yüksekkaya, "Decay and blow up of solutions for a delayed wave equation with variable-exponents," $C$ POST, vol. 3, no. 1, pp. 91-96, 2020.

[14] E. Pişkin and H. Yüksekkaya, "Local existence and blow up of solutions for a logarithmic nonlinear viscoelastic wave equation with delay," Computational Methods for Differential Equations, vol. 9, no. 2, p. 623636, 2021.

[15] E. Pişkin and H. Yüksekkaya, "Nonexistence of global solutions of a delayed wave equation with variable-exponents," Miskolc Mathematical Notes1-19.
[16] E. Pişkin and H. Y. Ksekkaya, "Decay of solutions for a nonlinear Petrovsky equation with delay term and variable exponents," The Aligarh Bull. of Maths, vol. 39, no. 2, pp. 63-78, 2020.

[17] F. Li, "Global existence and blow-up of solutionsfor a higherorder kirchhoff-type equation with nonlinear dissipation," Applied Mathematics Letters, vol. 17, no. 12, pp. 1409-1414, 2004.

[18] S. A. Messaoudi and B. S. Houari, "A blow-up result for a higher-order nonlinear Kirchho/-type hyperbolic equation," Applied Mathematics Letters, vol. 20, pp. 866-871, 2007.

[19] E. Pişkin and N. Polat, "On the decay of solutions for a nonlinear higher-order Kirchhoff-type hyperbolic equation," JARAM, vol. 5, no. 2, pp. 107-116, 2013.

[20] V. Georgiev and G. Todorova, "Existence of a solution of the wave equation with nonlinear damping and source terms," Journal of Partial Differential Equations, vol. 109, no. 2, pp. 295-308, 1994.

[21] E. Vitillaro, "Global nonexistence theorems for a class of evolution equations with dissipation," Archive for Rational Mechanics and Analysis, vol. 149, no. 2, pp. 155-182, 1999.

[22] M. Ohta, "Blow-up of solutions of dissipative nonlinear wave equations," Hokkaido Mathematical Journal, vol. 26, pp. 115-124, 1997.

[23] M. Ohta, "Remarks on blow-up of solutions for nonlinear evolution equations of second order," Advances in Mathematical Sciences and Applications, vol. 8, pp. 901-910, 1998.

[24] S. A. Messaoudi, "Global existence and nonexistence in a system of Petrovsky," Journal of Mathematical Analysis and Applications, vol. 265, no. 2, pp. 296-308, 2002.

[25] W. Chen and Y. Zhou, "Global nonexistence for a semilinear Petrovsky equation," Nonlinear Anal., vol. 70, no. 9, pp. 3203-3208, 2009.

[26] E. Pişkin and N. Polat, "On the decay of solutions for a nonlinear Petrovsky equation," Mathematical Sciences Letters, vol. 3, no. 1, pp. 43-47, 2013.

[27] T. Matsuyama and R. Ikehata, "On global solutions and energy decay for the wave equations of Kirchho/ type with nonlinear damping terms," Journal of Mathematical Analysis and Applications, vol. 204, pp. 729-753, 1996.

[28] K. Ono, "On global existence, asymptotic stability and blowing up of solutions for some degenerate nonlinear wave equations of Kirchhoff type," Mathematical Methods in the Applied Sciences, vol. 20, pp. 151-177, 1997.

[29] K. Ono, "Global existence, decay and blow up of solutions for some mildly degenerate nonlinear Kirchho/ strings," Journal of Difference Equations, vol. 137, 301 pages, 1997.

[30] K. Ono, "On global solutions and blow-up of solutions of nonlinear Kirchhoff strings with nonlinear dissipation," Journal of Mathematical Analysis and Applications, vol. 216, pp. 321342, 1997.

[31] A. Benaissa and S. A. Messaoudi, "Blow-up of solutions of a quasilinear wave equation with nonlinear dissipation," Journal of Partial Differential Equations, vol. 15, pp. 61-67, 2002.

[32] S. T. Wu and L. Y. Tsai, "Blow-up of solutions for some nonlinear wave equations of Kirchhoff type with some dissipation," Nonlinear Analysis: Theory, Methods \& Applications, vol. 65, no. 2, pp. 243-264, 2006.

[33] Y. Ye, "Global existence of solutions and energy decay for a Kirchhoff-type equation with nonlinear dissipation," Journal of Inequalities and Applications, vol. 2013, no. 1, 2013. 
[34] Y. Ye, "Global existence and energy decay estimate of solutions for a higher-order Kirchhoff type equation with damping and source term," Nonlinear Analysis: Real World Applications, vol. 14, no. 6, pp. 2059-2067, 2013.

[35] Q. Gao, F. Li, and Y. Wang, "Blow-up of the solution for higher-order Kirchhoff-type equations with nonlinear dissipation," Central European Journal of Mathematics, vol. 9, no. 3, pp. 686-698, 2011.

[36] H. A. Levine, "Instability and nonexistence of global solutions to nonlinear wave equations of the form $P u_{t t}=A u+F(u)$," Transactions of the American Mathematical Society, vol. 192, pp. 1-21, 1974.

[37] H. A. Levine, "Some additional remarks on the nonexistence of global solutions to nonlinear wave equations," SIAM Journal on Mathematical Analysis, vol. 5, no. 1, pp. 138-146, 1974.

[38] H. A. Levine, S. R. Park, and J. Serrin, "Global existence and global nonexistence of solutions of the Cauchy problem for a nonlinearly damped wave equation," Journal of Mathematical Analysis and Applications, vol. 228, no. 1, pp. 181-205, 1998.

[39] H. A. Levine, P. Pucci, and J. Serrin, "Some remarks on global nonexistence for nonautonomous abstract evolution equations," Communications in Contemporary Mathematics, vol. 208, pp. 253-263, 1997.

[40] H. A. Levine and J. Serrin, "Global nonexistence theorems for quasilinear evolution equations with dissipation," Archive for Rational Mechanics and Analysis, vol. 137, no. 4, pp. 341361, 1997.

[41] E. Pişkin and N. Polat, "Global existence, exponential decay and blow-up of solutions for coupled a class of nonlinear higher-order wave equations," General Mathematics Notes, vol. 8, no. 2, pp. 34-48, 2012.

[42] E. Pişkin and N. Polat, "Global existence, exponential and polynomial decay solutions for a system class of nonlinear higher-order wave equations with damping and source terms," International Journal of Pure and Applied Mathematics, vol. 76, no. 4, pp. 559-570, 2012.

[43] E. Pişkin and N. Polat, "Global existence and exponential decay of solutions for a class of system of nonlinear higherorder wave equations with strong damping," JARAM, vol. 4, no. 4, pp. 26-36, 2012.

[44] Y. Ye, "Global Existence and asymptotic behavior of solutions for Some nonlinear Hyperbolic equation," Journal of Inequalities and Applications, vol. 2010, no. 1, Article ID 895121, 2010.

[45] J. Zhou, X. Wang, X. Song, and C. Mu, "Global existence and blowup of solutions for a class of nonlinear higher-order wave equations," Zeitschrift für Angewandte Mathematik und Physik, vol. 63, no. 3, pp. 461-473, 2012.

[46] R. A. Adams and J. J. F. Fournier, Sobolev Spaces, Academic Press, 2003.

[47] E. Pişkin, Sobolev Spaces, Se kin Publishing, 2017.

[48] M. Nakao, "Asymptotic stability of the bounded or almost periodic solution of the wave equation with nonlinear dissipative term," Journal of Mathematical Analysis and Applications, vol. 58, no. 2, pp. 336-343, 1977.

[49] S. A. Messaoudi, "Blow up of positive-initial-energy solutions of a nonlinear viscoelastic hyperbolic equation," Journal of Mathematical Analysis and Applications, vol. 320, pp. 902915, 2006. 